\title{
Effects of technogenic pollutants on chicken embryos
}

\author{
Sabrie Zinabadinova, Viktoria LaVrinenko, Rostislav Kaminsky, \\ Alina Korsak, Liudmyla Sokurenko, Yuri Chaikovsky
}

O.O. Bogomolets National Medical University, Department of Histology and Embryology, T. Shevchenko boulevard 13, 01601 Kyiv, Ukraine

\section{ARTICLE INFO}

Received 13 July 2017

Accepted 30 October 2017

\section{Keywords:}

asbestos,

carbon black,

chicken embryo,

yolk sac.

\begin{abstract}
Increasing interest in nanomaterials can be explained by their broad involvement in many fields of industry and medicine. It is known that carbon black and crysotile asbestos behave like nanosized objects. They are wide spread in environment and have close contact with living organisms. The aim of our study was to define the effects of carbon black and crysotile asbestos on structures of the developing organism.

We used chicken embryos as a model for our study. Total amount of 77 eggs was divided in three groups: one control and two experimental with introduced carbon black and asbestos respectively. Researched materials were injected in the yolk on the third day of incubation and tissues for study were taken during early, middle and late stages of incubation. Histological and electron microscopy methods were used.

Carbon black and asbestos introduction caused underdevelopment of embryos and vessels depletion in blood islands of the yolk sac. Asbestos slowed down angiogenesis. Soot particles caused integrity violation of vessels and led to extravasation. The manifestations of soot influence were damages to blood circulatory system and structural disorders on cellular level. Introduction of chrysotile asbestos mostly resulted in compensatoryadaptive reactions like increasing in hematopoiesis and transcytosis in endotheliocytes.
\end{abstract}

\section{INTRODUCTION}

Asbestos fibers and carbon black (soot) are widely known technogenic pollutants with well-studied biological effects on the respiratory system and skin [1,2]. However, we decided to pay attention to their influence on the whole organism as they include nanosized components that might have high penetration ability and cause biotoxicity [3-6]. Asbestos and soot were even included into the category of antropogenic-unintentional nanomaterials [7]. Recently a new direction in the field of nanomaterials biocompatibility emerged - nanoparticles embryotoxicity $[8,9]$. In particular, the apparent structural link (similarity of fiber form) between carbon nanowires and asbestos fibers has generated serious doubts about their safety profile.

On the $26^{\text {th }}$ of June 2017, an order banning the use and manufacturing of products from all types of asbestos was issued in Ukraine. However, according to industry representatives, the projection of mortality data in European countries, where amphibole asbestos was used, to Ukraine, which has always used chrysotile asbestos, is incorrect. "The Ukrainian Chrysotile Association" appealed to the Ministry

\footnotetext{
* Corresponding author

e-mail: 1-sokurenko@i.ua
}

of Health with a request to recall the ban order, because these are different types of asbestos, which differ in their structure and properties, and have different risk for human health. Thus, the study of the influence of different types of asbestos is an urgent problem for our country and for the world as a whole. The issues touched upon in our work are due to the need to deepen knowledge about the effects of asbestos fibers on the body.

Therefore, our aim was to define the effects of carbon black and crysotile asbestos on structures of the developing organism, on tissue and cellular levels. We used chicken embryos as a test-system. This being said, we want to emphasize that in natural conditions they will not collide with the specified particles. But thanks to their isolation and autonomy, they are ideally suited for studying the reactions of tissues and organs to injected substances.

\section{MATERIALS AND METHODS}

Chicken embryos obtained from High Line eggs have been used as research model. The substances were injected as a suspension based on biocompatible dextran plasma substitute (Rheopolyglucinum). All the eggs were divided into 
three groups: the control (CG): 31 eggs injected with a suspension of biocompatible dextran; the experimental group EG-C: 19 eggs injected with a suspension of chrysotile asbestos; the experimental group EG-CB: 27 eggs injected with a suspension of carbon black. The dosage of substances injected to each egg was $14 \mathrm{mg}$ per $1 \mathrm{~kg}$ of weight (calculated according to the common medical practice of determining a single maintenance dose of charcoal in conversion to the weight of egg yolk). The material has been sterilized at $120^{\circ} \mathrm{C}$ for 60 minutes. Asbestos and soot are known for their flame retardant properties, i.e. they do not collapse under the influence of high temperatures. Therefore, these materials can withstand the use of rigid sterilization.

Asbestos crumb (K-6-30) was obtained from asbestostechnical products in the laboratory of the Institute of Surface Chemistry after A.A. Chuiko (NAS of Ukraine).

The soot was obtained as a combustion product of carbon-containing materials in the laboratory of the Institute of Surface Chemistry after A.A. Chuiko (NAS of Ukraine).

We studied embryos at early (from 3 up to 8 days), middle (from 9 up to 15 days) and late (from 16 up to 21 days) stages of incubation. Herein, electron microscopy was used as a complex method to study the ultrastructure of the chicken embryo cells. Various tissues chosen for electron microscopy were excised, cut into small chunks, and fixed for 1 to 4 hours by immersion in $2 \%$ glutaraldehyde in $0.1 \mathrm{M}$ cacodylate buffer (CB, pH 7.4). After washing out the fixatives with $0.1 \mathrm{M} \mathrm{CB}$, the chunks were treated with $2 \%$ osmium tetroxide $\left(\mathrm{OsO}_{4}\right)-0.1 \mathrm{M} \mathrm{CB}$ (a mixture of $2 \%$ $\mathrm{OsO}_{4}+0.1 \mathrm{M}$ phosphate buffer). The tissues were then dehydrated in ascending concentrations of ethanol, infiltrated with propyleneoxide, and embedded in Epon. Semi-thin sections $(1-1.5 \mu \mathrm{m}$ thick) and ultra-thin sections $(50-60 \mathrm{~nm})$ were produced by ultramicrotome UMTP - 6M (Ukraine) and contrasted with uranylacetate and lead nitrate according to Reynolds (1975). Samples were studied and photographed under the electron microscope JEM 100 CX (JEON, Japan).

\section{RESULTS}

Histological study. The yolk sac of the control group embryos revealed the formation of the appropriate number of blood islands, with endoderm cells of the yolk sack being histologically similar to adipocytes. They were large in size, had rounded shape and had peripherally located nucleus, while their inner contents were represented by huge vacuoles that made the cytoplasm look foamy (Fig. 1). The size of yolk inclusions in the cells depended on the degree of processing of the yolk surrounding endoderm cells. As a rule, at the early stages of yolk processing, the cells had one huge yolk inclusion, occupying almost the entire area of the cell. At the same time, a sufficient amount of unprocessed yolk was observed outside the cell. At later stages of yolk assimilation (as evidenced by insignificant accumulation of this material in microscope field of view), signs of remarkable compartmentalisation were observed in the internal contents of endodermal cells. Small (secondary) blood "satellite islands" formed around the central island vessel, with active haematopoiesis taking place in them. At the same time, the wall of the "satellite islands" was represented by just a single layer of endothelio-like cells, while in the primary central vessel smooth muscle cells and components of connective tissue could already be seen at this stage (Fig. 1).

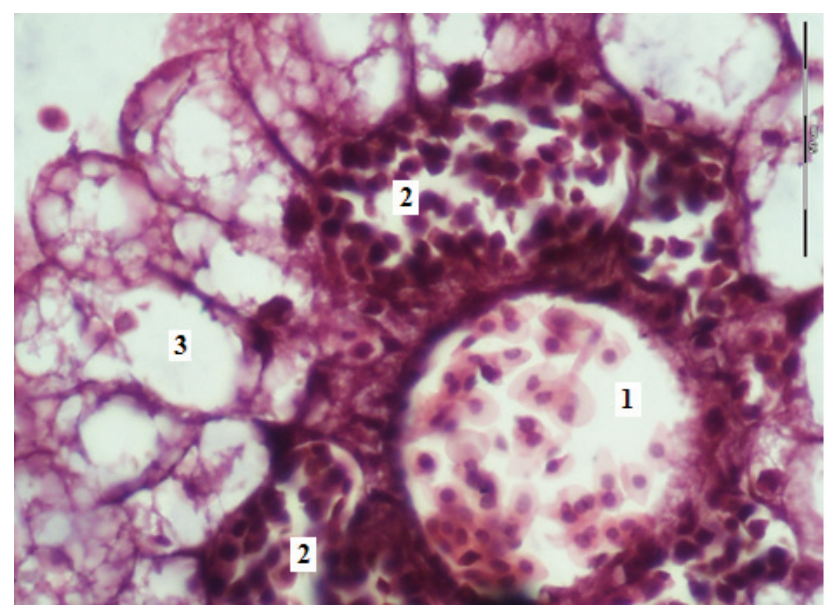

1 - central vessel, 2 - "satellite islands", 3 - endodermocytes

Figure 1. The formed central vessel of blood island in the yolk sac of control group embryo on day 10 of incubation. Hematoxylin and eosin staining $\times 600$

The embryos injected with chrysotile asbestos revealed almost no blood islands of small diameter (Fig. 2C). On the contrary, islands of large diameter with impaired formation of blood vessels were seen (Fig. 2D). Under such conditions, the existing "satellite islands" of the central vein played an important role in hemopoiesis. However, when depleted, they were unable to ensure an appropriate level of hemopoesis. In addition, the yolk sac vessels became empty and collapsed.

Unlike the influence of asbestos, impaired integrity of the blood vessels walls in the yolk sac blood islands resulted from the treatment of embryos with soot, which was confirmed by multiple intra-membrane extravasations (Fig. 2E). Moreover, intravascular aggregation of haemocytes was observed (Fig. 2F). A characteristic feature registered for the embryos from this group was focal infiltration of soot injected in the yolk to the cytomembrane of the cells of yolk sac. This coloured the latter in a specific grey-black colour. What is more, there was almost no uptake of yolk granules or their permeation through the wall of the yolk sac vessels. This effect argues for some delay in embryo development.

In the electron microscopy of the yolk sac cells, the introduction of chrysotile asbestos caused some disorders in endoderm cells metabolism, with formation of several medium-sized vacuoles being one of characteristic features (Fig. 2). As in the control group, we observed one big yellow vacuole, where processing and division of its contents to separate qualitative compounds took place. Here, the observed division of granules in the experimental group, in our opinion, demonstrated the weakening of the enzyme system in the endoderm cells.

The ultrastructural study of blood islands cells of the yolk sac has shown the endoderm cells to have smaller size and few yolk granules in their cytoplasm (Fig. 3). This also evidences substantial delay in embryo development. The endodermocytes having yolk granules in the cytoplasm were 


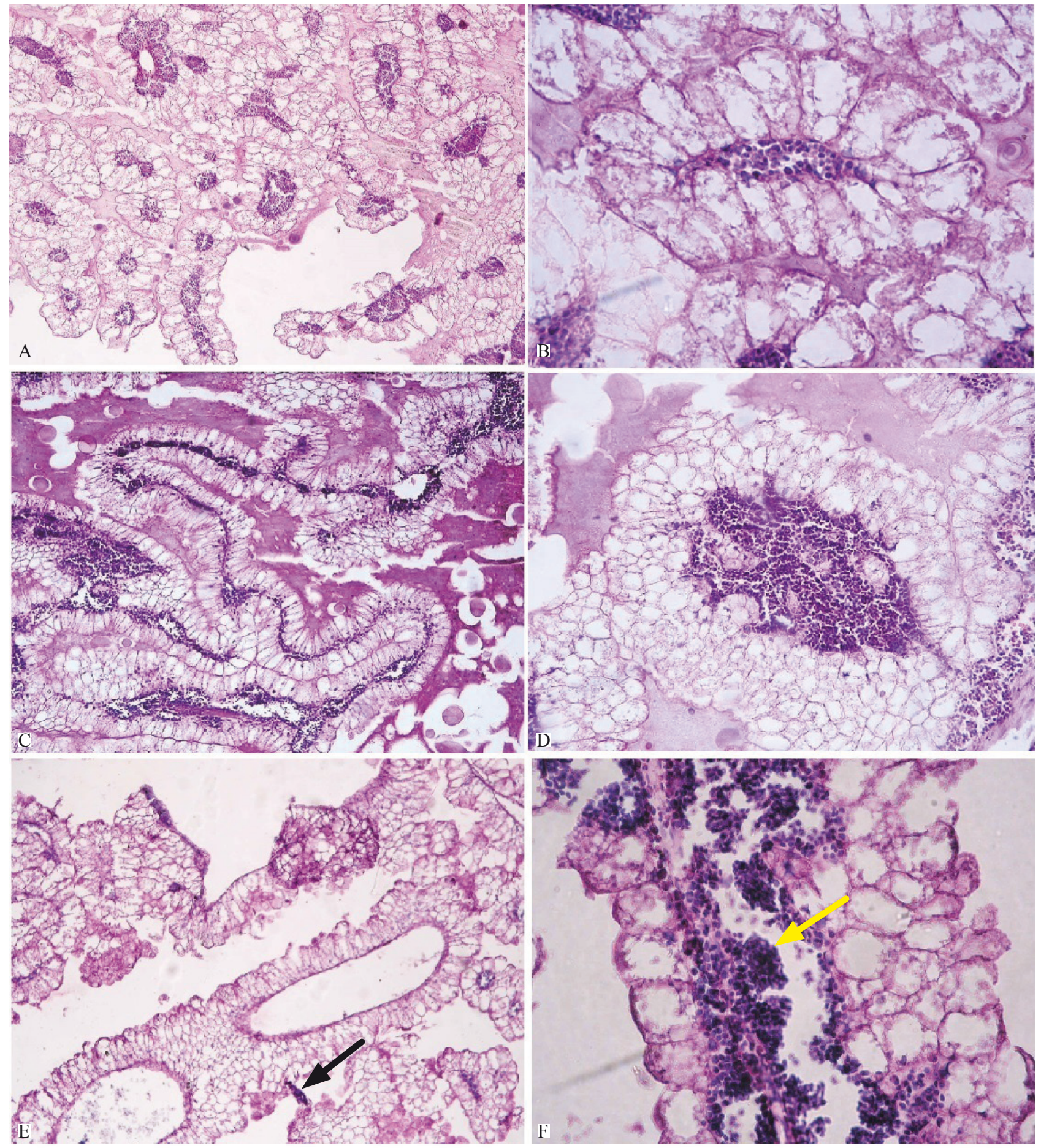

Figure 2. Blood island in the yolk sac of 14 day embryos from the control group (A, B), from the chrysotile introduced group (C, D) and from the soot introduced group (E, F). Black arrow - soot particles, yellow arrow - intravascular aggregation of haemocytes. Hematoxylin and eosin staining; A, C, E - ×100, B, F - ×400, D - ×200

characterised by inclusion of highly condensed material in the granules that most probably were soot (Fig. 3). In the blood islands of embryos with introduced chrysotile, there was an intensified transcytosis in the endotheliocytes; vacuoles of large diameter could be found, as well as an increased number of outgrowths of the plasma membrane on the vascular surface. We often observed mitotic figures in the cells of hematopoietic islands, hence, no remarkable disorders in the proliferation processes of hematopoietic blood cells were registered. In the histological research section, we have described the "satellite islands" that surrounded the central vessel and were formed in the islands of middle and large size. Ultrastructural research has demonstrated that there are certain "nurse" cells morphologically similar to such type of cells in thymus. "Nurse cells" formed compartments where hematopoietic cells were developing (Fig. 4A). 


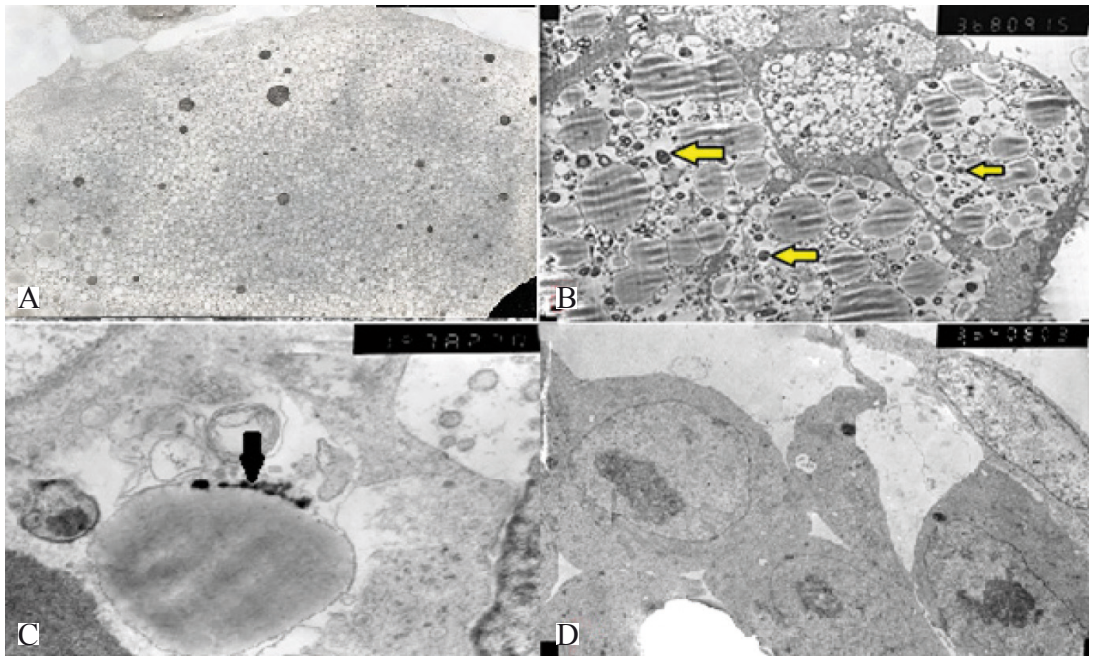

Figure 3. Endoderm cells of embryo yolk sac. A - endoderm cell of the 6-day embryo from the control group; B - endoderm cell of the 6-day embryo from the chrysotile introduced group (arrows - yolk granules); C - concentration of high electron density material (most probably - soot) (arrow) on the edge of the lipid vacuole in the yolk sac cell of the 6-day embryo from the soot introduced group, D - endoderm cells without yolk granules of the 3-day embryo from the soot introduced group. A: $\times 4800, B, D: \times 3600$, C: $\times 19000$

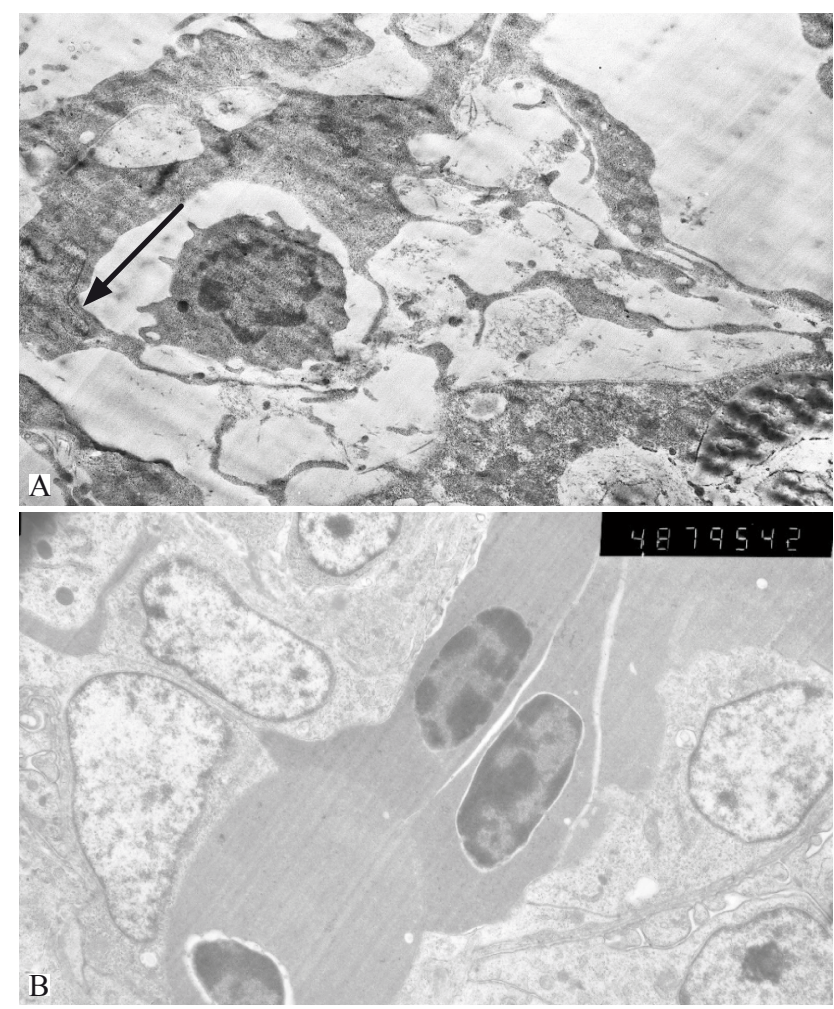

Figure 4. Developing hematopoietic cell surrounded by outgrowths of nurse-cell (black arrow) of the 6-day embryo from the chrysotileintroduced group. $\times 3600$ (A). Aggregation of erythrocytes in the vessel of the yolk sac of 20-day embryo introduced with soot. $\times 4800(\mathrm{~B})$

Even though active proliferation processes took place in "satellite islands", the thickness of membrane between them and central vessel was bigger than in newly formed small islands. Such a barrier might have prevented the formed cells from fast involvement into blood circulation. Thus, we believe that even enhanced haematopoiesis in the islands of medium and large diameter could not induce appropriate compensatory function.
Endotheliocytes of soot-introduced embryos also showed signs of pathology. There was local oedema in the cytoplasm, the mitochondria were characterised by vacuolisation and cristolisis, and rough endoplasmic reticulum channels were remarkably broadened. According to the results of electron microscopic study, soot penetrated the vessels of the embryo membranes and caused aggregation of erythrocytes in the blood stream. On the surface and in the cytoplasm of erythrocytes, we found material of high electron density that most probably is soot (Fig. 4B).

\section{DISCUSSION}

Normal embryonic development is highly dependent on the proper functioning of the yolk sac that provides transport through the endodermal layer, as well as metabolization of maternal macromolecules such as the layer of mesoderm which produces the first blood cells within blood islands $[10,11]$. The result of our research broadened the data on the histological structure of blood islands. We think that this is the key to understanding of the role of the yolk sac in trophic function and haematopoiesis in chicken embryo. In our work, we showed that the size of yolk inclusions in the cells depended on the degree of processing of the yolk surrounding endoderm cells. We also added to world knowledge several details about the interaction of blood islands structural parts. It was already known that there are central vessels in the middle of blood islands with active haematopoiesis taking place in them [10]. We revealed that small (secondary) blood "satellite islands" form around these central island vessels. The wall of the "satellite islands" is formed by a single layer of endothelial-like cells, while in the primary central vessel, smooth muscle cells and components of connective tissue could already be seen at this stage.

The embryos injected with chrysotile asbestos revealed almost no blood islands of small diameter. On the contrary, there were islands of large diameter with impaired blood vessel formation. We hold that the newly formed (small) islands were the basis for the enlargement and branching of the network of the yolk sac blood vessels which underpins the normal embryogenesis. Therefore, the mentioned feature demonstrates a slowing down of yolk utilisation by EG-C embryos, resulting in developmental disorders within them. Macroscopic analysis of embryos from this group has demonstrated a large amount of unutilized yolk, constriction of the yolk sac vessels without any visible pathology of their walls and delayed embryo development which support the above. It is noteworthy that low blood content in the yolk sac vessels was also observed in embryos treated with carbon nanofibers [12]. Furthermore, the application of carbon nanofibers inhibited the angiogenesis of the chorioallantoic membrane in chicken embryos [12]. That is why we assume the similar effects of asbestos fibers and carbon nanofibers based on their apparent structural link. 
An ultrastructural study of the blood islands cells of the yolk sac after the introduction of chrysotile asbestos has shown the endoderm cells to have smaller size and few yolk granules in their cytoplasm - hence that evidences substantial delay in embryo development.

Intensified transcytosis in the endotheliocytes, vacuoles of large diameter and increased number of outgrowths of plasma membrane on the vascular surface are considered to be manifestation of compensatory reaction in the course of inadequate formation of new hematopoiesis islands in the yolk sac.

Unlike the influence of asbestos, impaired integrity of the blood vessels walls in the yolk sac blood islands resulted from the treatment of embryos with soot. This was confirmed by the presence of multiple intra-membrane extravasations. What is more, there was almost no uptake of yolk granules or their permeation through the wall of the yolk sac vessels. This argues for some delay in embryo development. According to the latest microscopic researches nanodiamonds generate resembling alteration effects in chicken embryo. The toxic effects of asbestos fibers and carbon black particles were also investigated on hepatocytes [13,14]. Herein, morphological abnormalities such as different types of degeneration were revealed. Hence, we have summarized the obvious biological effects of both types of particles on exchange processes in cells with high level of metabolism.

Using chicken embryos as a test-system allowed a more detailed research of the pattern of spreading, as well as an enabling of the biological effects of the substances in question. The fact that chicken embryo develops in the environment almost completely isolated from the external influence minimizes the impact of exogenous factors on the course of the experiment, thus enabling the ongoing monitoring of physiological and pathological processes. Such work offers some organizational and economic benefits and allows an evaluation of the bodily reaction to these materials in a large number of cell populations.In summation, the submitted material broadens the current knowledge about the biological effects of some of the most well-known and common technogenic pollutants (asbestos and soot) by evaluating their effects on cellular and subcellular levels.

\section{CONCLUSIONS}

Introduction of asbestos and soot to the yolk sac of chicken embryos caused delay in their development.

Asbestos slowed down angiogenesis. The influence of asbestos mostly resulted in compensatory-adaptive reactions (intensification of hematopoiesis and transcytosis in endotheliocytes).
Particles of soot caused integrity damage of vessels, effusions of blood and aggregation of erythrocytes. The main manifestations of soot influence on cellular level were signs of the vacuolisation of the cytoplasm and organelles.

\section{REFERENCES}

1. Baur X., Wilken D. Effect of asbestos fiber dust exposures on lung function--a systematic review. Pneumologie. 2010; 64 (2): 81-110.

2. Saputra D., Yoon J.H., Park H., Heo Y., Yang H., Lee E.J. et al. Inhalation of carbon black nanoparticles aggravates pulmonary inflammation in mice. Toxicol Res. 2014; 30 (2): 83-90.

3. Barua S., Mitragotri S. Challenges associated with Penetration of Nanoparticles across Cell and Tissue Barriers: A Review of Current Status and Future Prospects. Nano Today. 2014; 9 (2): 223-243.

4. Funahashi S., Okazaki Y., Ito D., Asakawa A., Nagai H., Tajima M. et al. Asbestos and multi-walled carbon nanotubes generate distinct oxidative responses in inflammatory cells. J Clin Biochem Nutr. 2015; 56 (2): 111-17.

5. Kaminsky F. Rostislav, Sokurenko M. Liudmyla, Chaikovsky B. Yuri Status of rats myocardium under subchronic mercury exposure and its pharmacological correction Curr Issues Pharm Med Sci., 2016; 29(4): 167-70

6. Omelchuk S., Aleksijchuk V., Sokurenko L., Blagaia A., Prudchenko S. Characteristics of rat liver exposed to nanoparticles of lead compounds. Georgian Med News. 2016 Des;(261):94-9.

7. Oberdorster G., Oberdorster E., Oberdorster J. Nanotoxicology: an emerging discipline evolving from studies of ultrafine particles. Environ Health Perspect. 2005; 113 (7): 823-39.

8. Al Moustafa A., Mfoumou E., Roman D.E., Nerguizian V., Alazzam A., Stiharu I. et al. Impact of single-walled carbon nanotubes on the embryo: a brief review. Int J Nanomedicine. 2016; 11: 349-355.

9. Celá P, Veselá B., Matalová E., Večeřa Z., Buchtová M.Embryonic toxicity of nanoparticles. Cells Tissues Organs. 2014; 199 (1): 1-23.

10. Fernandes R., Costola-Souza C., Sarmento C., Gonçalves L., Favaron Ph. O., Miglino M. A. Placental tissues as sources of stem cells. Open Journal of Animal Sciences. 2012; 2: 166-173.

11. Guedes P., Oliveira B., Manso P., Caputo L., Cotta-Pereira G., PelajoMachado M. Histological analyses demonstrate the temporary contribution of yolk sac, liver, and bone marrow to hematopoiesis during chicken development. PLoS One. 2014; 9(3).

12. Lavrinenko V.E., Zinabadinova S.S., Shobat L.B., Sokurenko L.M., Chaikovsky Yu.B. Influence of nanodiamonds and carbon nanowires on survival and cells structure in chicken embryo. Georgian Medical News. 2016; 6 (255): 93-9.

13. Zinabadinova S.S. Features of structural and functional conditions of chiken embryo liver which are caused by asbestos fibers and carbon nanowires. Bulletin of problems in biology and medicine. 2015; 2/1 (118): 265-9.

14. Lavrinenko V. E. Biological effect of nanodiamonds and soot on structural and functional conditions of chiken embryo liver and kidneys. Bulletin of problems in biology and medicine. 2015; 2/1 (118): 269-73. 